\title{
Automation of anaesthesia: a review on multivariable control
}

\begin{abstract}
Anaesthesia is a multivariable problem where a combination of drugs are used to induce desired hypnotic, analgesia and immobility states. The automation of anaesthesia may improve the safety and cost-effectiveness of anaesthesia. However, the realization of a safe and reliable multivariable closed-loop control of anaesthesia is yet to be achieved due to a manifold of challenges. In this paper, several significant challenges in automation of anaesthesia are discussed, namely model uncertainty, controlled variables, closed-loop application and dependability. The increasingly reliable measurement device, robust and adaptive controller, and better fault tolerance strategy are paving the way for automation of anaesthesia.
\end{abstract}

Keyword: Closed-loop anaesthesia; Model uncertainty; Fault-tolerant; MIMO; Depth of anaesthesia 